\title{
Yearning and its Measurement in Complicated Grief
}

Donald J. Robinaugh ${ }^{1,2}$, Christine Mauro ${ }^{3}$, Eric Bui ${ }^{2}$, Lauren Stone $^{2}$, Riva Shah ${ }^{2}$, Yuanjia Wang $^{3}$, Natalia A. Skritskaya ${ }^{4}$, Charles F. Reynolds ${ }^{5}$, Sidney Zisook ${ }^{6}$, Mary-Frances O’Connor ${ }^{7}$, Katherine Shear ${ }^{4}, \&$ Naomi M. Simon ${ }^{2}$

${ }^{1}$ Department of Psychology, Harvard University

${ }^{2}$ Massachusetts General Hospital, Department of Psychiatry

${ }^{3}$ Columbia University, Mailman School of Public Health (Department of Biostatistics)

${ }^{4}$ Columbia University, School of Social Work

${ }^{5}$ University of Pittsburgh, Department of Psychiatry

${ }^{6}$ University of California San Diego and San Diego VA Healthcare System

${ }^{7}$ University of Arizona, Department of Psychology

Author Note

Correspondence concerning this article should be addressed to Donald J. Robinaugh, Department of Psychiatry, Massachusetts General Hospital, 1 Bowdoin, MA 02114. E-mail: drobinaugh@partners.org. 
Donald J. Robinaugh is a Clinical Fellow in Psychology in the Massachusetts General Hospital, Department of Psychiatry. His research interests are focused on understanding and treating emotional disorders that develop following loss and trauma.

Christine Mauro is an Associate Research Scientist in the Department of Biostatistics at Columbia University. Her expertise is in the analysis of clinical trials, psychometrics, and longitudinal data analysis.

Eric Bui is the Associate Director for Research at the Center for Anxiety and Traumatic Stress Studies and Complicated Grief Program at the Massachusetts General Hospital and is an Assistant Professor of Psychiatry at Harvard Medical School. His research interests are focused on the etiology and treatment of anxiety and stress-related conditions.

Lauren Stone is a Human Evolutionary Biology major at Harvard College and research assistant at Massachusetts General Hospital, Department of Psychiatry. She is researching the neurological bases of chronic stress.

Riva Shah is a medical student at Rush University and a former clinical research coordinator at the Massachusetts General Hospital, Department of Psychiatry. Her research interests include bereavement, trauma, anxiety disorders, and addiction.

Yuanjia Wang is an Associate Professor of Biostatistics at Columbia University, and is an expert in statistical analyses of clinical trial and observational study data.

Natalia A. Skritskaya is an Associate Research Scientist at Columbia School of Social Work. She also is a licensed clinical psychologist and works primarily with bereaved individuals. Her research interests include complicated grief, empirically supported treatments and their dissemination.

Sidney Zisook is a distinguished professor of psychiatry at the University of California San Diego. His research interests include bereavement, suicide prevention, and the treatment of major depressive disorders.

Charles F. Reynolds is an Endowed Professor in Geriatric Psychiatry at the University of Pittsburgh Medical Center and Director of the Aging Institute at the University of Pittsburgh. His primary research interests focus on mood, grief, and sleep disorders of later life.

Mary-Frances O'Connor is an Assistant Professor in the Psychology Department at the University of Arizona. She studies the impact of grief and loss on psychological and physiological functioning.

Katherine Shear is the Director of the Center for Complicated Grief at Columbia School of Social Work and is the Marion E. Kenworthy Professor of Psychiatry at the Columbia School of Social Work and Columbia University College of Physicians and Surgeons. Dr. Shear is a leading expert in complicated grief and is the developer of Complicated Grief Therapy. 
Naomi M. Simon is the Director of the Center for Anxiety and Traumatic Stress Disorders at Massachusetts General Hospital and Associate Professor of Psychiatry at Harvard Medical School. Dr. Simon's major clinical and research interests include initial and next step treatments for anxiety disorders, anxiety comorbid with mood disorders, complicated grief, and the biological impact of chronic stress due to these disorders. 


\begin{abstract}
Persistent intense yearning for the deceased is a core clinical feature of complicated grief (CG) that distinguishes it from other mental disorders that develop following loss. The Yearning in Situations of Loss Scale (YSL) is a recently developed assessment of yearning. To assess the psychometric properties of the YSL in those with CG, we administered the YSL, Inventory of Complicated Grief, and Quick Inventory of Depression Symptomatology to 303 treatmentseeking bereaved adults with CG. Our results suggest the YSL is a reliable assessment with acceptable convergent and discriminate validity as a measure of yearning in those with CG.
\end{abstract}

Keywords: Complicated Grief, Bereavement, Yearning, Longing, Assessment 


\section{Yearning and its Measurement in Complicated Grief}

Yearning is an unsatisfied, intense, and future-oriented appetitive desire (Davis, 1984). It is not merely missing something from the past (i.e., being aware of its absence), but rather entails actively desiring something in the future. Importantly, yearning is experienced in reference to an object. One yearns for something. The central role of the desired object in yearning suggests that it is best conceptualized as an "affectively charged cognitive event" (Kavanagh, Andrade, \& May, 2005, p. 447) with the cognitive representation of the desired object and yearning-related negative affect combining to produce the subjective experience of yearning.

Yearning for the presence of a deceased loved one is a core symptom of complicated grief (CG; Shear et al., 2011), a bereavement-specific syndrome characterized by prolonged, distressing, and impairing grief. Grief researchers have emphasized the importance of yearning in this syndrome, both because it is the most commonly reported grief-related affective experience following loss (Maciejewski, Zhang, Block, \& Prigerson, 2007; Robinaugh, LeBlanc, Vuletich, \& McNally, 2014) and because it distinguishes CG from other mental disorders that develop following the death of a loved one, such as depression or post-traumatic stress disorder (Prigerson, Frank, et al., 1995).

\section{Measuring Yearning in Bereaved Adults}

Despite the prominent role of yearning in grief and CG, there is relatively little research examining yearning in bereaved adults. As of January $8^{\text {th }} 2015$, there were 58 English-language peer-reviewed empirical articles indexed in Psych Info under any combination of the terms "grief", "complicated grief", or "bereavement" with the terms "yearning" or "longing". Of these, only 15 included a sample of bereaved adults and reported a quantitative assessment of yearning. Most commonly, yearning was assessed by a single-item drawn from a grief or complicated grief 
symptom inventory (e.g., "I feel myself longing for the person who died..."; Prigerson, Frank, et al., 1995). Although there are limitations to single-item measurement (Diamantopoulos, Sarstedt, Fuchs, Wilczynski, \& Kaiser, 2012), these single-item assessments of yearning have the advantage of being high in representational accuracy. That is, as direct queries of a relatively specific construct, single-item measures correspond closely to the construct they are designed to assess (McGrath, 2005). Much of what has been reported about the prevalence (e.g., Horowitz et al., 1997), trajectory, (e.g., Maciejewski et al., 2007), and neurobiology of yearning in CG (O'Connor et al., 2008) has relied on these single-item assessments.

A second common assessment of yearning is from 6 publications that used data from the Changing Lives of Older Couples (CLOC) study (e.g., Stroebe, Abakoumkin, \& Stroebe, 2010). In the CLOC study, researchers created a yearning subscale using 4-items from their assessment of grief. These items assess a range of affective experiences, including yearning for the deceased, missing the deceased, emotional pain, and loneliness. The variety of affective experiences included in this subscale may contribute to its relatively low internal consistency $(\alpha=.68)$ and raises questions about its representational accuracy as a measure of yearning. Indeed, in the most recently proposed diagnostic criteria for CG (American Psychiatric American Psychiatric Association, 2013), yearning, emotional pain, and feelings of loneliness are each identified as distinct symptoms of CG. Accordingly, the yearning subscale used in the CLOC study may better be considered an assessment of several core emotional experiences in grief, rather than an assessment of yearning alone.

The Yearning in Situations of Loss Scale. More recently, O'Connor and Sussman developed the Yearning in Situations of Loss scale (YSL; O'Connor \& Sussman, 2014) to assess yearning across situations of loss (e.g., the death of a loved, romantic separation, and prolonged 
separation from home). In creating this scale, the authors drew on clinical interviews and theoretical work on yearning to generate an initial 32-item scale before removing 11 items with low item-scale correlations, resulting in the 21-item YSL. In contrast to commonly used singleitem assessments of yearning, the YSL does not assess yearning by directly querying its presence. Indeed, the word yearning is not used in any of the YSL items. The majority of items in the YSL query cognitions. In particular, the YSL includes numerous items assessing the imagination of a counterfactual reality in which the deceased did not pass away, including items tapping experiential simulation and evaluative counterfactual thought (Markman \& McMullen, 2003). Experiential simulation (cf. elaborated intrusions; Kavanagh et al., 2005, pp. 447-449) denotes vividly imagining the counter-factual future as though it were real (e.g., YSL Item \#11: "I like to imagine what I would do if [the deceased] were with me"). Evaluative counterfactual thought refers to cognitions in which the counter-factual reality is contrasted with the current reality. In the YSL, several items implicitly and unfavorably contrast the current reality without the deceased with the counterfactual reality in which the deceased is present (e.g., YSL Item \#20: "I feel like if [the deceased] were here, I would know what to do about things."). Accordingly, the YSL appears to assess yearning largely by assessing the cognitive component of this affectively laden cognitive event.

In their preliminary examination of the resultant 21-item YSL, O’Connor and Sussman (2014) found that it exhibited excellent reliability $(\alpha=.94)$ and good discriminant and convergent validity in individuals who had experienced the death of a loved one, romantic separation, or prolonged separation from home. Of particular interest to the current study, the YSL exhibited a large correlation, $r(158)=.70$, with complicated grief symptom severity (as 
measured by the Inventory of Complicated Grief; Prigerson, Frank, et al., 1995) in the subset of the sample who had experienced the death of a loved one.

\section{Current Study}

In this study, we extend these initial findings on the YSL by examining the factor structure, reliability, and validity of the YSL among a large sample of treatment-seeking bereaved adults with clinically assessed and formally diagnosed CG. To our knowledge, this study is the first to assess the psychometric properties of the YSL in a clinical sample of treatment-seeking bereaved adults adults formally diagnosed with CG. To assess convergent validity, we examine the associations among the YSL, a commonly used single-item assessment of yearning, and a measure of CG symptom severity. To examine discriminant validity, we will assess the associations between the YSL and depression symptom severity, a syndrome that commonly arises following the death of a loved one but does not entail yearning for the deceased. Because yearning is not a component of depression, the YSL should exhibit stronger associations with overall CG symptom severity than with depression. In addition to these primary aims, we conduct exploratory analyses to determine whether YSL scores are related to characteristics of the loss, characteristics of the relationship with the deceased, or demographic characteristics of the bereaved.

\section{Method}

\section{Participants}

Participants were treatment-seeking bereaved adults $(n=303)$ who participated in a multi-site trial examining the efficacy of a grief focused psychotherapy, and the selective serotonin reuptake inhibitor citalopram (Shear, 2015). Participants were required to have experienced the loss at least 6 months prior to enrollment $(M=4.7$ years, $S D=7.4 ; M d n=2.2$ 
years; range $=1-59$ years), score at least 30 on the Inventory of Complicated Grief (ICG;

Prigerson, Maciejewski, et al., 1995), report that CG was their primary problem during a clinical assessment, and be judged on clinical interview to suffer from CG. In addition, for the present study, participants must have completed the YSL. The sample was predominately female (77.9\%) and Caucasian (79.9\%). The mean age was 52.9 years. The most common relationships to the deceased were spouse or significant other (36.6\%), parent $(29.0 \%)$, child (18.8\%), and sibling $(9.6 \%)$. The most common causes of death were long-term illness (i.e., greater than one month, $43.8 \%)$, short-term illness (20.5\%), suicide (13.5\%), accident (14.5\%), and murder $(4.3 \%)$.

All data for this study were taken from baseline assessments. The Institutional Review Boards of Partners Healthcare, Columbia University, the University of California San Diego, and the Pittsburgh School of Medicine approved the protocols and consent forms for these studies. Participants provided written informed consent.

\section{Measures}

Yearning in Situations of Loss (YSL). The YSL (O'Connor \& Sussman, 2014) is a 21item measure of yearning and its cognitive and affective correlates. Participants rated items on a 5-point scale from Never to Always in reference to the deceased loved one whose death the participant identified as being the most upsetting. Total scores range from 21 to 105, with higher scores indicating greater yearning.

Self-report measures of psychopathology. The Inventory of Complicated Grief (ICG; Prigerson, Maciejewski, et al., 1995; $\alpha=.75$ ) is a 19-item measure assessing complicated grief symptoms. All participants in this study met or exceeded a score of 30 on the ICG; a cut-off commonly employed to indicate the presence of CG (Shear, Frank, Houck, \& Reynolds, 2005). 
The fourth item of the ICG (i.e., "I feel myself longing for the person who died...") is a commonly used single-item assessment of yearning and is reported in the results below using the name "ICG-Yearning." Because this item could artificially inflate the association between yearning and overall complicated grief symptom severity, we omitted it when calculating the ICG total scores unless otherwise noted.

In addition, we administered the Quick Inventory of Depressive Symptomatology - Self Report (QIDS-SR; Rush et al., 2003) to assess symptoms of depression. The QIDS-SR is a 16item self-report assessment in which participants rate 9 symptoms of depression on a 4-point scale ranging from Not Present to Severe. Scores range from 0 to 27, with scores of 6, 11, and 16 indicating mild, moderate, and severe depression, respectively.

\section{Results}

\section{Psychometric Properties}

YSL scores ranged broadly from 27 to $105(M=75.73, M d n=78.00$, Mode $=89.00, S D$ $=16.36)$. There was a moderate negative skew to the distribution of scores (skewness $=-.54, S E$ skewness = .14). We used IBM SPSS Statistics for Windows (v22) to perform an iterated exploratory factor analysis (EFA) with maximum likelihood estimation and direct oblimin rotation. The EFA produced 3 factors with initial eigenvalues exceeding the Kaiser criterion (9.38, 1.55, 1.27; Kaiser, 1960). The inflexion point of the Scree plot (Cattell, 1966) occurred at the $2^{\text {nd }}$ eigenvalue. Accordingly, we evaluated one-, two-, and three-factor solutions. Relative to the two-factor and three-factor solutions, the one-factor solution produced a more clearly interpretable pattern of findings. All items loaded highly on this factor and it accounted for $44.68 \%$ of the total variance. The results of the one-factor solution appear in Table 1. 
YSL scores exhibited excellent internal consistency $(\alpha=.94)$. The corrected item-total correlations and squared multiple correlations for each item using the full sample appear in Table 1. Each item exhibited a moderate to large corrected item-total correlation, $r s>.47$. There were no items whose deletion would improve internal consistency.

The results for convergent and discriminant validity appear in Table 2 . There was a large correlation between YSL scores and the single-item assessment of yearning drawn from the ICG $r(301)=.55,95 \%$ CI $[.48, .63], p<.001$ and a large correlation with ICG total scores, $r(301)=$ $.51,95 \%$ CI $[.42, .59], p<.001$. YSL scores were more strongly associated with ICG scores than QIDS scores, $r(295)=.34,95 \%$ CI $[.22, .44], p<.001$, findings which support the discriminate validity of the YSL scores.

\section{Correlates of Yearning in Complicated Grief}

Finally, we examined whether YSL scores were related to demographic characteristics of the bereaved, characteristics of the loss, or the nature of the relationship to the deceased. Oneway ANOVAs revealed no main effect of gender, $F(1,301)=0.05, p=.829$, race, $F(5,297)=$ $1.11, p=.357$, or cause of death, $F(5,295)=0.73, p=.598$. There was a small positive association between YSL scores and age, $r(301)=.14,95 \% \mathrm{CI}[.03, .25], p=.018$ and a small negative association between YSL scores and time since loss, $r(301)=-.14,95 \%$ CI [-.23,-.05], $p=.016$. There was also a significant main effect of relationship to the deceased on YSL scores, $F(4,296)=5.33, p<.001$. Tukey's HSD post-hoc tests revealed that those who experienced spousal loss $(M=79.77, S D=15.35)$ reported higher YSL scores relative to those who lost a parent $(M=72.97, S D=14.84)$, sibling $(M=70.24, S D=17.77)$, or “other" relation $(M=66.06$, $S D=18.49), p s<.05$. Those who experienced the death of a child $(M=78.60, S D=16.23)$ did not differ significantly from those experiencing any other type of loss. 


\section{Discussion}

To our knowledge, this study is the first to assess the psychometric properties of YSL scores in a sample of adults with CG. Our results suggest that the YSL is a reliable assessment in bereaved adults with $\mathrm{CG}$ and exploratory factor analyses suggest that a single factor best fit the data. Notably, eleven of the thirteen items that loaded highest on the YSL factor assess evaluative counterfactual thought in which the participants unfavorably contrasted their present reality with a counterfactual reality that includes the deceased. Accordingly, the YSL appears to assess yearning principally, though not exclusively, by measuring the cognitive representation of the desired object (i.e., a preferred counterfactual reality in which the deceased is present).

Our results provide some support for the validity of YSL scores as an assessment of yearning in those with CG. It exhibited large correlations with a commonly used single-item assessment of yearning and was more strongly associated with overall CG symptom severity than with depression severity. These findings parallel those reported by O'Connor and Sussman (2014) in their sample of bereaved adults with and without complicated grief. In that study, researchers similarly found that a one-factor solution best fit the data and that YSL scores were more strongly associated with CG, $r(158)=.70$, than with other syndromes that commonly arise following loss, including depression, $r(158)=.50$, and anxiety, $r(158)=.48$. Although we observed a smaller association between YSL and ICG scores in our study than was reported by O'Connor and Sussman (2014), this diminished effect size is likely attributable to the restricted range of CG scores in our sample due to the inclusion criteria for the broader study.

There is one caveat to our broader conclusion that YSL scores are a valid assessment of yearning. The correlation between the YSL and the single-item assessment of yearning was roughly equivalent to the correlation between the YSL and our measure of CG. If the YSL 
measures yearning, we would expect it to exhibit stronger correlation with other assessments of yearning than with measures of related constructs, such as CG. It may be the case that the YSL assesses a broader syndrome rather than yearning alone. Consistent with this possibility, several YSL items inquire about affective experiences distinct from yearning (e.g., loneliness in item 17 and anhedonia or emotional numbness in item 15). Moreover, the focus in the YSL on cognitions related to the deceased suggests YSL scores may also reflect preoccupation with thoughts related to the deceased, another distinct symptom of CG. Accordingly, caution is warranted in concluding that the YSL measures yearning alone and not a broader CG-related cluster of symptoms.

In addition to examining the psychometric properties of YSL scores, we examined whether YSL scores are related to characteristics of the loss, characteristics of the relation to the deceased, or demographic characteristics of the bereaved. YSL scores did not vary by gender, race, or cause of death. There was a negative association between YSL scores and time since loss, suggesting that yearning may subside over time, even among those with CG. There was also an effect of relationship to the deceased on YSL scores. Individuals who experienced spousal loss exhibited higher YSL scores than all other participants except those who experienced the loss of a child. There was not a statistically significant difference in YSL scores between those who lost a spouse and those who lost a child. These findings suggest that yearning may be an especially important part of the CG syndrome in those who experienced the death of a spouse.

\section{Recommendations for the Assessment of Yearning}

For researchers interested in assessing yearning in CG, the YSL is the most comprehensive and psychometrically sound assessment of yearning currently available. However, our findings also suggest that a commonly used single-item assessment of yearning 
exhibited strong correlations with total YSL scores and, thus, may be an acceptable alternative if a more concise assessment is required.

The YSL may also be useful in clinical settings as it will allow clinicians to assess the individual thoughts and feelings that constitute yearning and, thereby, obtain a richer conceptualization of the patient's subjective experience of this prominent feature of CG. This additional information can help guide treatment by identifying thoughts that fuel the subjective experience of yearning and, thus, are likely to be fruitful targets of intervention.

\section{Conclusion}

In this study, YSL scores exhibited excellent reliability as well as acceptable convergent and discriminant validity in a sample of bereaved adults with CG. Accordingly, our findings suggest that the YSL is a useful addition to the battery of assessments targeting grief-related thoughts, feelings, and behaviors in those with CG. 


\section{References}

American Psychiatric Association. (2013). Diagnostic and statistical manual of mental disorders (5th ed.). Arlington, VA: American Psychiatric Association.

Cattell, R. B. (1966). The scree test for the number of factors. Multivariate Behavioral Research, $1,245-276$.

Davis, W. A. (1984). The two senses of desire. Philisophical Studies, 45, 181-195.

Diamantopoulos, A., Sarstedt, M., Fuchs, C., Wilczynski, P., \& Kaiser, S. (2012). Guidelines for choosing between multi-item and single-item scales for construct measurement: a predictive validity perspective. Journal of the Academy of Marketing Science, 40(3), 434449. doi: 10.1007/s11747-011-0300-3

Horowitz, M. J., Siegel, B., Holen, A., Bonanno, G. A., Milbrath, C., \& Stinson, C. H. (1997). Diagnostic criteria for complicated grief disorder. American Journal of Psychiatry, 154(7), 904-910. doi: http://dx.doi.org/10.1176/ajp.154.7.904

Kaiser, H. F. (1960). The application of electronic computers to factor analysis. Educational and Psychological Measurement, 10, 141-151.

Kavanagh, D. J., Andrade, J., \& May, J. (2005). Imaginary relish and exquisite torture: The elaborated intrusion theory of desire. Psychological Review, 112(2), 446-467. doi: 10.1037/0033-295x.112.2.446

Maciejewski, P. K., Zhang, B., Block, S. D., \& Prigerson, H. G. (2007). Empirical examination of the stage theory of grief. Journal of the American Medical Association, 297(7), 716723. 
Markman, K. D., \& McMullen, M. N. (2003). A reflection and evaluation model of comparative thinking. Personality and Social Psychology Review, 7(3), 244-267. doi: 10.1207/S15327957pspr0703_04

McGrath, R. E. (2005). Conceptual complexity and construct validity. Journal of Personality Assessment, 85(2), 112-124. doi: 10.1207/s15327752jpa8502_02

O'Connor, M. F., \& Sussman, T. J. (2014). Developing the yearning in situations of loss scale: Convergent and discriminant validity for bereavement, romantic breakup, and homesickness. Death Studies, 38(7), 450-458. doi: 10.1080/07481187.2013.782928

O'Connor, M. F., Wellisch, D. K., Stanton, A. L., Eisenberger, N. I., Irwin, M. R., \& Lieberman, M. D. (2008). Craving love? Enduring grief activates brain's reward center. Neuroimage, 42(2), 969-972. doi: 10.1016/j.neuroimage.2008.04.256

Prigerson, H. G., Frank, E., Kası, S. V., Reynolds, C. F., Anderson, B., Zubenko, G. S., . . . Kupfer, D. J. (1995). Complicated grief and bereavement-related depression as distinct disorders: Preliminary empirical validation in elderly bereaved spouses. American Journal of Psychiatry, 152(1), 22-30. doi: http://dx.doi.org/10.1176/ajp.152.1.22

Prigerson, H. G., Maciejewski, P. K., Reynolds, C. F., Bierhals, A. J., Newsom, J. T., Fasiczka, A., ... Miller, M. (1995). Inventory of complicated grief: A scale to measure maladaptive symptoms of loss. Psychiatry Research, 59(1-2), 65-79. doi: 10.1016/01651781(95)02757-2

Robinaugh, D. J., LeBlanc, N. J., Vuletich, H. A., \& McNally, R. J. (2014). Network analysis of persistent complex bereavement disorder in conjugally bereaved adults. Journal of Abnormal Psychology, 123(3), 510-522. doi: 10.1037/abn0000002 
Rush, A. J., Trivedi, M. H., Ibrahim, H. M., Carmody, T. J., Arnow, B., Klein, D. N., . . Keller, M. B. (2003). The 16-Item Quick Inventory of Depressive Symptomatology (QIDS), clinician rating (QIDS-C), and self-report (QIDS-SR): a psychometric evaluation in patients with chronic major depression. Biological Psychiatry, 54(5), 573-583. doi: S0006322302018668

Shear, M. K., Frank, E., Houck, P. R., \& Reynolds, C. F., 3rd. (2005). Treatment of complicated grief: a randomized controlled trial. Journal of the American Medical Association, 293(21), 2601-2608. doi: 10.1001/jama.293.21.2601

Shear, M. K., Simon, N., Wall, M., Zisook, S., Neimeyer, R., Duan, N., . . Keshaviah, A. (2011). Complicated grief and related bereavement issues for DSM-5. Depression \& Anxiety, 28(2), 103-117. doi: 10.1002/da.20780

Shear, M. K. (2015, May). Psychotherapy for complicated grief: An evidence-based approach. In Shear, M. K. (2015), Optimizing treatment of complicated grief: Results of a multicenter clinical trial. Symposium conducted at the $168^{\text {th }}$ Annual Meeting of the American Psychiatric Association, Toronto, Canada.

Stroebe, W., Abakoumkin, G., \& Stroebe, M. (2010). Beyond depression: Yearning for the loss of a loved one. Omega-Journal of Death and Dying, 61(2), 85-101. doi: 10.2190/Om.61.2.A 
Table 1

Reliability and Exploratory Factor Analysis Results

\begin{tabular}{cccc} 
& & \multicolumn{2}{c}{ Reliability } \\
\cline { 3 - 4 }$\%$ & Corrected & Squared \\
$\%$ & Factor & Item-Total & Multiple
\end{tabular}

YSL Item

$M$ (SD) Endorsed Loading Correlation Correlation

19 It's hard to imagine feeling as comfortable and happy as I was when I was with

10 The feeling of wanting __ back is so strong it is indescribable.

5 It's hard for me to be happy without -

21 I feel like I could be completely happy if I was with _.

2 I find myself wishing that things could be the way they were when I was with

15 I feel like it would be impossible to find another person that would make me feel the same way as __ did.

9 I feel separate from the world around me without

17 Without _, I feel alone.

18 I don't feel like going out as much without _.

20 I feel like if _ were here, I would know what to do about things.

14 I wish I could do the things I used to do with

$3.65(1.21) \quad 57 \%$

$57 \% \quad .81$

.78

.66

$\begin{array}{llll}3.54(1.30) & 54 \% & .79 & .77\end{array}$

.64

$\begin{array}{llll}3.71(1.06) & 60 \% & .75 & .71\end{array}$

.61

$3.41(1.29) \quad 51 \%$

.73

.72

.70

.64

$4.10(1.02)$

$74 \%$

.73

$3.89(1.28)$

$66 \%$

.71

.69

.60

$3.37(1.18) \quad 48 \% \quad .71$

.69

.68

.65

$3.86(1.12) \quad 68 \%$

.66

.62

$\begin{array}{lll}3.24(1.32) & 45 \% & .66 \\ 3.69(1.16) & 58 \% & .66\end{array}$

.64

.60
16 I miss _ as much or more than I have missed any other person.

13 I feel that there just is no one else who can love me the way _ did.

11 I like to imagine what I would do if _ were with me.

4 I daydream about ..

$3.98(1.03) \quad 69 \% \quad .64$

$4.51(0.88) \quad 88 \% \quad .64$

.62

.61

.47

$3.70(1.39) \quad 61 \% \quad .61$

.57

.54

$\begin{array}{llll}3.63(1.12) & 58 \% & .59 & .58\end{array}$

.57

.56

.57

.57

.61

.54

.56

6 I feel like things used to be so perfect before I lost_..

8 I imagine and choose things I would be willing to give

$3.18(1.03)$

$40 \%$

.58

$3.09(1.36) \quad 40 \% \quad .58$

$3.10(1.47) \quad 43 \% \quad .58$

.55

.53

.56

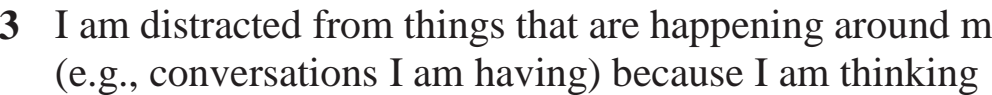
about

7 I feel that in my ideal world, losing _ would never have happened.

1 I am reminded of _ by everyday objects, places and occurrences.

12 I am much more engaged in a conversation if it is about.

$\begin{array}{llll}4.08(1.21) & 72 \% & .53 & .51 \\ 4.05(0.92) & 72 \% & .51 & .48 \\ 3.01(1.32) & 36 \% & .47 & .47\end{array}$

.51

Note. YSL $=$ Yearning in Situations of Loss Scale. Items appear in decreasing order by the magnitude of their factor loading. Endorsement was defined as reporting a score greater than 3 for that item (i.e., endorsing it more than "sometimes"). 
Table 2

Correlations among yearning, complicated grief, post-traumatic stress disorder, and depression

\begin{tabular}{lccc|c|cc}
\hline & $M$ & $S D$ & $\alpha$ & ICG-Yearning & ICG $^{a}$ & QIDS-SR \\
\hline YSL & 75.73 & 16.36 & .94 & $.55[.46, .63]^{* * *}$ & $.51[.42, .59]^{* * *}$ & $.34[.23, .44]^{* * *}$ \\
ICG-Yearning & 3.33 & .75 & -- & -- & $.43[.33, .52]^{* * *}$ & $.14[.04, .25]^{*}$ \\
\hline ICG $^{a}$ & 42.00 & 8.44 & .73 & & -- & $.37[.28, .46]^{* * *}$ \\
QIDS & 13.43 & 4.27 & -- & & & -- \\
\hline
\end{tabular}

Note. YSL = Yearning in Situations of Loss Scale, ICG = Inventory of Complicated Grief, ICGYearning = Inventory of Complicated Grief Item 4, QIDS-SR = Quick Inventory of Depression Symptomatology - Self Report. The 95\% confidence interval for each correlation was calcualted using bootstrapping with 1,000 samples. The sample size for each correlation varied between 297 and 303 due to missing data.

${ }^{a}$ In this table, we report the mean, standard deviation, reliability, and correlations for the ICG total scores with the yearning item removed. The full ICG total score was associated with the YSL, $r(301)=.54,95 \%$ CI [.44,.62], $p<.001$.

${ }^{*} p<.05,{ }^{* * *} p<.001$ 